Annals of Mathematics, 148 (1998), 1153-1154

\title{
Counterexample to boundary regularity of a strongly pseudoconvex CR submanifold: An addendum to the paper of Harvey-Lawson
}

\author{
By Hing Sun Luk and Stephen S.-T. YAu*
}

The purpose of this paper is to give a counterexample of Theorem 10.4 in [Ha-La]. In the Harvey-Lawson paper, a global result is claimed, but only a local result is proven. This theorem has had a big impact on CR geometry for almost a quarter of a century because one can use the theory of isolated singularities to study the theory of CR manifolds and vice versa.

Example. Consider the following holomorphic map:

$$
\begin{aligned}
F: \mathbf{C}^{2} & \longrightarrow \mathbf{C}^{3} \\
(u, v) & \longrightarrow(x, y, z)=\left(u(u-1), v, u^{2}(u-1)\right) .
\end{aligned}
$$

Clearly for any $c, F$ restricted on the line $\{v=c\}$ is an embedding outside the two points $(0, c)$ and $(1, c) . F$ sends $(0, t)$ and $(1, t)$ to $(0, t, 0)$ for all $t$. Now take $S$, which is the boundary of a ball $B=\left\{(u, v) \in \mathbf{C}^{2}:\|(u, v)\| \leq 2\right\}$. It is easy to see that the mapping $F$ restricted on $S$ is still an embedding. The image of $S$ under $F$ is a strongly pseudoconvex $\mathrm{CR}$ manifold in $\mathbf{C}^{3}$. The variety that $F(S)$ bounds is $F(B)$. Observe that $F(B)$ has curve singularities along the line $(0, t, 0)$. We remark that $F\left(\mathbf{C}^{2}\right)$ is a hypersurface $\{(x, y, z) \in$ $\left.\mathbf{C}^{3}: z^{2}-z x-x^{3}=0\right\}$ in $\mathbf{C}^{3}$.

Theorem 10.4 of [Ha-La] was so powerful that it has been used by many researchers. Fortunately, we can replace it by the following theorem, the proof of which will appear elsewhere [Lu-Ya].

Theorem. Let $X$ be a strongly pseudoconvex CR manifold of dimension $2 n-1, n \geq 2$. If $X$ is contained in the boundary of a bounded strictly pseudoconvex domain $D$ in $\mathbf{C}^{N}$, then there exists a complex analytic subvariety $V$ of dimension $n$ in $D-X$ such that the boundary of $V$ is $X$. Moreover, $V$ has boundary regularity at every point of $X$, and $V$ has only isolated singularities in $V \mid X$.

\footnotetext{
*Yau's research supported by NSF. Luk's research partially supported by RGC Hong Kong.
} 
Acknowledgement. We thank Professor Lempert who first suggested to us that Theorem 10.4 of [Ha-La] may be wrong. In fact it was Lempert who first told the second author a concrete geometric description of how to construct a counterexample to the boundary regularity theorem of Harvey-Lawson (Theorem 10.4 of [Ha-La]) in the higher codimension case. His ideas were realized by two simple examples by us in [Lu-Ya].

Department of Mathematics, The Chinese University of Hong Kong, Shatin, N.T., Hong Kong

E-mail address: hsluk@math.cuhk.edu.hk

Department of Mathematics, Statistics, and Computer Science, University of Illinois at Chicago, 851 S. Morgan Street, Chicago, IL, 60607-7045

E-mail address: yau@uic.edu

\section{REFERENCES}

[Ha-La] F. R. Harvey and H. B. Lawson, Jr., On boundaries of complex analytic varieties, I, Ann. of Math. 102 (1975), 223-290.

[Lu-Ya] H.-S. Luk and S. S.-T. Yau, Kohn-Rossi cohomology, holomorphic de Rham cohomology and the complex plateau problem, preprint.

(Received May 20, 1997) 